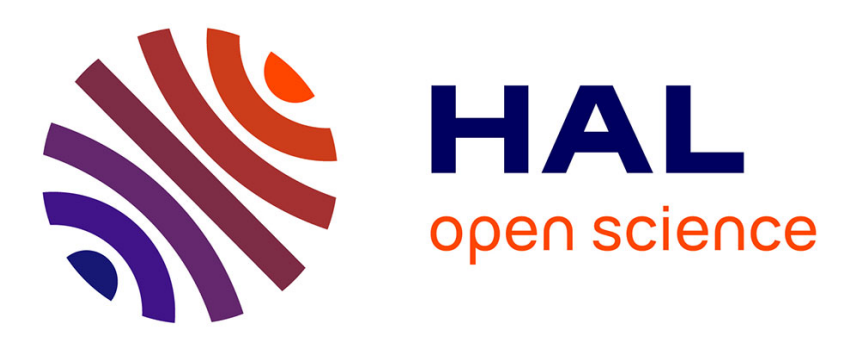

\title{
A 3D shock computational strategy for real assembly and shock attenuator
}

Hervé Lemoussu, Pierre-Alain Boucard, Pierre Ladevèze

\section{To cite this version:}

Hervé Lemoussu, Pierre-Alain Boucard, Pierre Ladevèze. A 3D shock computational strategy for real assembly and shock attenuator. Advances in Engineering Software, 2002, 33 (7-10), pp.517-526. 10.1016/S0965-9978(02)00074-1 . hal-01698044

\section{HAL Id: hal-01698044 https://hal.science/hal-01698044}

Submitted on 10 Sep 2019

HAL is a multi-disciplinary open access archive for the deposit and dissemination of scientific research documents, whether they are published or not. The documents may come from teaching and research institutions in France or abroad, or from public or private research centers.
L'archive ouverte pluridisciplinaire HAL, est destinée au dépôt et à la diffusion de documents scientifiques de niveau recherche, publiés ou non, émanant des établissements d'enseignement et de recherche français ou étrangers, des laboratoires publics ou privés. 


\title{
A 3D shock computational strategy for real assembly and shock attenuator
}

\author{
H. Lemoussu*, P.-A. Boucard, P. Ladevèze \\ LMT-Cachan, E.N.S. Cachan/C.N.R.S./Université Paris 6, 61 Avenue du Président Wilson, 94235 Cachan Cedex, France
}

\begin{abstract}
The extension of an approach, suitable for bolting structures impact computation with a large number of unilateral friction contact surfaces, and with local plasticity of the bolts, is presented. It is a modular approach based on a mixed domain decomposition method and the LATIN method. This iterative resolution process operates over the entire time-space domain. A 3D Finite-Element code is presented and dedicated to applications concerning connection refined models for which the structure components are assumed elastic. Several examples are analysed to show the method's capability of describing shocks throw real three-dimensional assembly. Comparisons between classical dynamic code LS-DYNA3D are presented.
\end{abstract}

Keywords: Impact; Frictional contact; plasticity; 3D-Computation

\section{Introduction}

The aim of the present work is to present the feasibility of a flexible computational tool that contains all the ingredients needed to represent the response of a real launcher assembly. The concerned loadings are associated with the shocks initiated by the explosion of a cord cutter on a spatial structure. This typical loading appears during the two-stage separation of a launcher. Therefore, this study is part of the pyrotechnic shock investigation conducted by the National Centre for Space Studies (CNES), based in Evry (France).

The goal is to be able to compute the behaviour of a real assembly in order to represent the evolution of a shock response spectrum that cross through an assembly or to have all the information needed to be able to build a dynamic condensed model of the assembly.

In order to meet this expectation, we use an approach well adapted to the problem to solve. This is leaded by the specificity of the problem:

- We are far enough from the shock initiation to admit that the contacting bodies remain in elasticity and satisfy the small perturbations hypothesis, except bolts which can plastify.

\footnotetext{
* Corresponding author. Tel.: + 33-1-47-40-21-86; fax: +33-1-47-40-2785.

E-mail address: herve.lemoussu@1mt.ens-cachan.fr (H. Lemoussu).
}

- The solution is characterised by the large number of friction contact surfaces that leads the structure into a highly non-linear global behaviour.

The principles of our approach are given in Ref. [7].

The first ingredient is a mixed-domain decomposition method that allows for a parallel-oriented analysis. The idea herein is to introduce a partition from two different mechanical entities. The structure is represented by an assembly of sub-structures and interfaces.

The sub-structures display a linear behaviour and the interfaces can display non-linear behaviour, exhibit to the friction contact problem or to bolt's plasticity. The unilateral contact with Coulomb friction is used and the plasticity is described by a simple classical model with one hardening variable.

The exchange between these two different types of substructure is performed with two quantities: velocity and force. This velocity-force-duality, is very well-suited with the dynamic framework.

To solve the mechanical problem associated with this decomposition, a computational strategy based on the LArge Time INcrement method (LATIN) [6,7], is proposed. Many works have demonstrated the ability of this approach to solve difficult problems, like large displacements, nonlinearity of material and contact problems [3] under a quasistatic hypothesis. This study reinforces such an approach and constitutes the final step in order to reach the response 


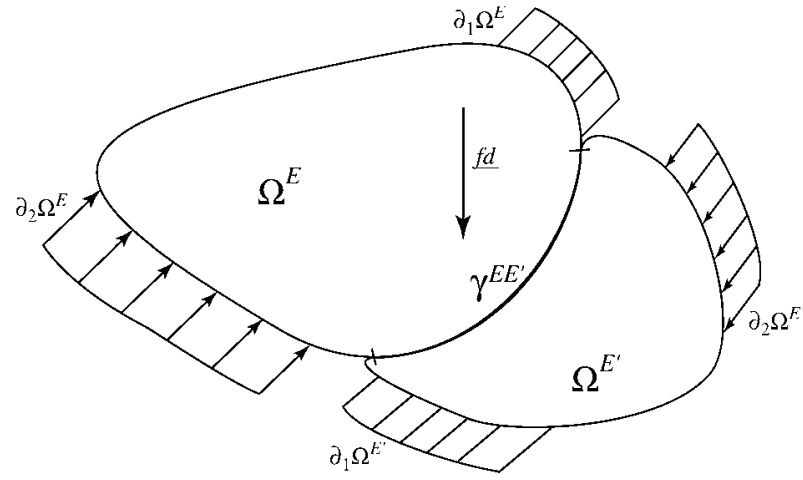

Fig. 1. Reference problem with contact.

of a real 3D-assembly using this dynamic extension to the LATIN method.

The proposed strategy is non-incremental and thus strongly differs from step-by-step methods, convergence mathematical results are proved in Ref. [7].

The unidimensional feasibility of this approach as been showed in previous work [2] and we describe herein its extension for 3D structures.

The basic principles of this approach are recalled and a full description of the method is provided within a dynamic three-dimensional framework. A specific strategy for solving, on the time-space domain, the global problem obtained at each iteration is detailed such as the specific treatment of contact conditions and plasticity, both taken into account at the interfaces.

This approach has been introduced into the finite element code DYGITA3D based on CASTEM 2000 [10]. Two examples will subsequently be used to highlight the feasibility of this method and its capability of describing shock and frictional unilateral contact problems with good convergence results. A comparison with the industrial code LS-DYNA3D [4] is developed.

\section{Reference problem}

The studied structure is $\Omega$ and the studied time interval is $[0, T]$. On a part $\partial_{1} \Omega$ of its boundary, a displacement or a velocity field can be prescribed. On the rest of the boundary $\partial_{2} \Omega$, traction boundary conditions can be applied (Fig. 1).

The structure can be made of several sub-structure $(\Omega=$ $\left.\Omega^{E}+\Omega^{E^{\prime}}\right)$, contacting together on an interface called $\gamma^{E E^{\prime}}$.

The problem is to find an element $(\underline{U}(\underline{M}), \boldsymbol{\sigma}(M))$ on $\Omega \times$ $[0, T]$ that satisfy:

- initial conditions:

$\forall \underline{M} \in \Omega, \quad\left\{\begin{array}{l}\underline{U}_{t=0}=\underline{U}_{0} \\ \underline{V}_{t=0}=\left.\cdot \frac{\mathrm{d} \underline{U}}{\mathrm{~d} t}\right|_{t=0}=\underline{V}_{0}\end{array}\right.$
- kinematic admissibility:

$\forall t \in[0, T], \quad\left\{\begin{array}{l}\underline{U}_{\partial_{1} \Omega}=\underline{U} d \\ \underline{U} \in \mathbf{U}^{[0, T]}\end{array}\right.$

- contact conditions:

$$
\begin{aligned}
& \forall t \in[0, T], \quad \forall \underline{M} \in \gamma^{E E^{\prime}}, \\
& \left\{\begin{array}{c}
U_{N}=U_{N}^{E^{\prime}}-U_{N}^{E} \geq 0 \\
F_{N}=F_{N}^{E}=-F_{N}^{E^{\prime}} \leq 0 \\
\left(U_{N}^{E}-U_{N}^{E^{\prime}}\right) F_{N}^{E}=0
\end{array}\right.
\end{aligned}
$$

- equilibrium equations:

$$
\begin{aligned}
& \forall t \in[0, T], \quad \forall \underline{U}^{*} \in \mathbf{U}_{0}, \\
& \int_{\Omega} \rho \frac{\mathrm{d}^{2} \underline{U}}{\mathrm{~d} t^{2}} \cdot \underline{U}^{*} \mathrm{~d} \Omega+\int_{\Omega} \operatorname{Tr}\left(\boldsymbol{\sigma} \varepsilon\left(\underline{U}^{*}\right)\right) \mathrm{d} \Omega \\
& =\int_{\Omega} \underline{f} d \cdot \underline{U}^{*} \mathrm{~d} \Omega+\int_{\partial_{2} \Omega} \underline{F} d \cdot \underline{U}^{*} \mathrm{~d} S
\end{aligned}
$$

- constitutive law:

$\forall t \in[0, T] ; \forall \underline{M} \in \Omega, \quad \boldsymbol{\sigma}(M, t)=\mathbf{K} \varepsilon(\underline{U})$

where $\mathbf{K}$ is the Hooke tensor, $\varepsilon(\underline{U})$ is the small displacement strain, $N$ is relevant to the normal vector of the interface, $\mathbf{U}^{[0, T]}$ is the space where the displacement $\underline{U}$, defined on $\Omega \times[0 ; T]$ is searched for. $\mathbf{U}_{0}$ is the virtual space defined by:

$$
\mathbf{U}_{0}=\left\{\underline{U}^{*} \cdot \underline{U}^{*}=0 \text { on } \partial_{1} \Omega, \text { regular }\right\}
$$

The resolution of this problem needs a space-time discretisation. The finite element method is frequently used [11] for the space domain and most of the numerical scheme can be written in the Newmark algorithm way [5,9]. In three-dimensional cases, the size of the problem to solve is not proportional to the number of degree of freedom (d.o.f.), but follows a power rule.

Therefore, many problems appear in terms of database manipulation and memory allocation and specific techniques are needed in order to be able to solve the problem. Therefore, domain decomposition methods are frequently used.

The main difference between these methods lies in the choice of the kinematic or dual quantities prescribed at the interface. 


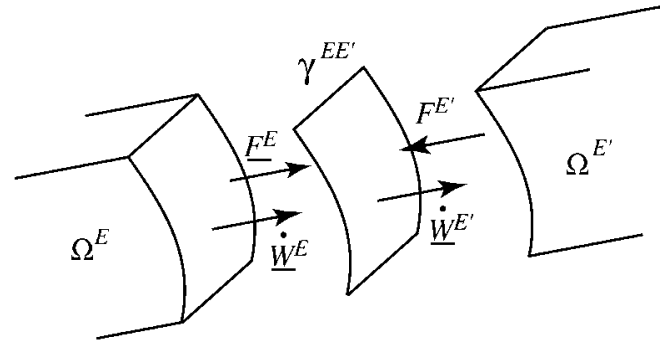

Fig. 2. Exchange between sub-structures and interfaces.

\section{Principles of the approach}

This approach is composed of two major ingredients:

- a mixed domain decomposition well adapted to the problem description,

- a specific resolution process using a non-incremental resolution technique.

\subsection{Structural decomposition}

The approach is slightly different from the classical methods. It is a real meso-modelisation of the structure that can be associated to the non-overlapping version of the Schwartz method [8].

The structure is decomposed into two mechanical entities: the substructures $\Omega^{E}$ and the interfaces $\gamma^{E E^{\prime}}$. Each of them has its own unknowns and its own equations. A substructure can dialogue with the interfaces around it, using both quantities: velocity and surface traction fields as it is shown in Fig. 2.

Moreover, this exchange dialogue existing between the two entities is mixed and performed with a velocity-force duality, which is very well-suited for the dynamic framework.

\subsection{Solution process}

In order to solve the problem associated with the above decomposition, we use a non-incremental approach, called the LATIN method, proposed by Ladevèze in 1985 (more details can be found in Ref. [7]). This method has yielded some excellent results for quasi-static loadings. Previous works have shown computation times divided by 50 for 3D

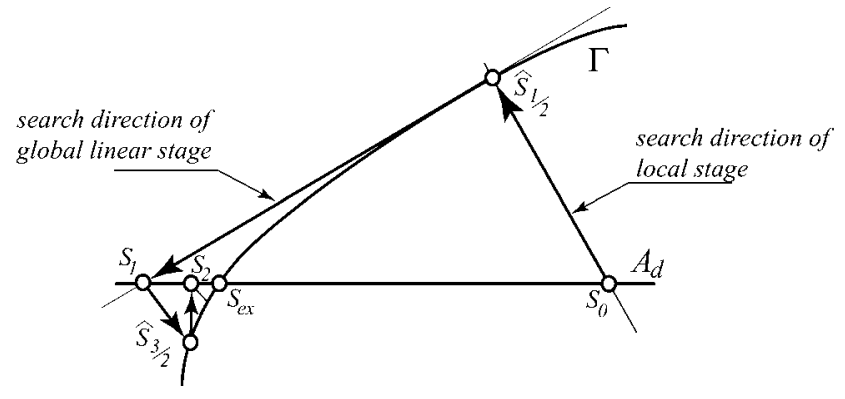

Fig. 3. Iterative scheme. connections problems with many contact surfaces [1]. The present study consists of its development in dynamics. Convergence mathematical results are proved in Ref. [7].

The LATIN method is based on three principles. The first one is to separate difficulties in order to avoid the simultaneity of global and non-linear problems. By taking into account the mechanical properties of the equations, two groups can be distinguished: the local space variables equations which might be non-linear on the one hand (the associated space will be called $\Gamma$ ), and the linear equations that might be global in space on the other hand (the associated space will be called $\mathbf{A} d$ ).

The second principle of the method is a two-stages iteration scheme which alternatively solves each set of equations. The local stage solves the problem associated with $\Gamma$ and leads to a non-unique solution; it is therefore necessary to add other equations, called search direction equations, $\left(E^{+}\right)$. The linear global stage solves the problem associated with $\mathbf{A} d$ and leads to an ill-posed problem. It becomes necessary to add new search direction equations, $\left(E^{-}\right)$. A representation of this scheme is given Fig. 3 where $s_{n}$ is the solution to a linear global stage and $\hat{s}_{n+1 / 2}$ is the solution to the local stage at iteration $n$.

The third principle of the method lies in the resolution of the global problem. One main characteristic of the present computational technique is that the global operators involved in this stage are constant with respect to the iterations. Thus, they are all treated during the method's initialisation.

\section{Resolution process}

Each iteration of our approach is composed of two stages.

\subsection{Global linear stage at iteration $n$}

The global linear stage begins with an element $\hat{s}_{n+1 / 2}\left(\hat{\dot{W}}_{n+1 / 2}^{E}, \hat{\dot{W}}_{n+1 / 2}^{E^{\prime}}, \hat{\hat{F}}_{n+1 / 2}^{E}, \hat{\hat{F}}_{n+1 / 2}^{E^{\prime}}\right)$ that belongs to $\Gamma$ and is defined on $\gamma^{E E^{\prime}} \times[0, T]$.

One then must find an element $s_{n+1}\left(\underline{V}_{n+1}^{E}, \boldsymbol{\sigma}_{n+1}^{E}\right)$, defined on $\Omega^{E} \times[0, T]$, that satisfies:

kinematic admissibility

initial conditions $\quad+$ search direction equations

equilibrium equations

$$
: \mid \begin{aligned}
& \underline{F}_{n+1}^{E}-\underline{\hat{F}}_{n+1 / 2}^{E}=-k_{0}\left(\underline{\dot{W}}_{n+1}^{E}-\underline{\hat{W}}_{n+1 / 2}^{E}\right) \\
& \underline{F}_{n+1}^{E^{\prime}}-\underline{\hat{F}}_{n+1}^{E^{\prime}}=-k_{0}\left(\underline{\dot{W}}_{n+1}^{E^{\prime}}-\underline{\hat{\dot{W}}}_{n+1 / 2}^{E^{\prime}}\right)
\end{aligned}
$$

where $E$ and $E^{\prime}$ are relative to sub-structures $\Omega^{E}$ and $\Omega^{E^{\prime}} . k_{0}$ is a positive constant and the method parameter associated with the global linear stage.

Using these search direction equations, the problem 
becomes:

$\forall t \in[0, T], \quad \forall \underline{V}^{*} \in \mathbf{V}_{0}^{E}$,

$$
\begin{aligned}
& \int_{\Omega^{E}} \rho \frac{\mathrm{d} \underline{V}_{n+1}^{E}}{\mathrm{~d} t} \cdot \underline{V}^{*} \mathrm{~d} \Omega+\int_{\Omega^{E}} \mathbf{K} \varepsilon\left(\underline{U}_{n+1}^{E}\right) \varepsilon(\underline{V}) \mathrm{d} \Omega+\int_{\partial \Omega^{E}} \\
& \times k_{0} \underline{V}_{n+1}^{E} \cdot \underline{V}^{*} \mathrm{~d} S \\
& =\int_{\Omega^{E}} \underline{f d^{E}} \cdot \underline{V}^{*} \mathrm{~d} \Omega+\int_{\partial \Omega^{E}}\left(\underline{\hat{F}}_{n+1 / 2}^{E}+k_{0} \underline{\hat{W}}_{n+1 / 2}^{E}\right) \cdot \underline{V}^{*} \mathrm{~d} S
\end{aligned}
$$

This is a classical formulation of a problem in which the density of surface traction $\underline{\tilde{F}}$ is applied:

$\underline{\tilde{F}}=\underline{\hat{F}}_{n+1 / 2}^{E}+k_{0} \underline{\hat{\dot{W}}}_{n+1 / 2}^{E}$

The added step herein concerns the resolution of the global problems, whereby the constancy of the matrices present throughout the iterations is used. Consequently, these matrices have been pre-calculated during the method's initialisation.

\subsection{Local stage at iteration $n$}

The local stage begins with a known element $s_{n}\left(\underline{V}_{n}^{E}, \sigma_{n}^{E}\right)$ that belongs to $\mathbf{A} d$ and is defined on $\Omega^{E} \times[0, T]$.

One then must find an element $\hat{s}_{n+1 / 2}\left(\hat{\dot{W}}_{n+1 / 2}^{E}, \underline{\hat{\dot{W}}}_{n+1 / 2}^{E^{\prime}}, \underline{\hat{F}}_{n+1 / 2}^{E}, \underline{\hat{F}}_{n+1 / 2}^{E^{\prime}}\right)$, defined on $\gamma^{E E^{\prime}} \times$ $[0, T]$, that satisfies:

linterface behaviour + search direction equations

$$
:\left\{\begin{array}{l}
\left.\hat{F}_{n+1 / 2}^{E}-\underline{F}_{n}^{E}\right)=k_{0}\left(\underline{\hat{W}}_{n+1 / 2}^{E}-\underline{\dot{W}}_{n}^{E}\right) \\
\left(\underline{\hat{F}}_{n+1 / 2}^{E^{\prime}}-\underline{F}_{n}^{E^{\prime}}\right)=k_{0}\left(\hat{\hat{W}}_{n+1 / 2}^{E^{\prime}}-\underline{\dot{W}}_{n}^{E^{\prime}}\right)
\end{array}\right.
$$

The resolution of this problem is greatly dependant of the involved type of interface. Two groups can be distinguished: linear behaviour (perfectly connected, boundary conditions, etc.) and non-linear behaviour (frictional contact with void and plasticity interfaces).

Frictional contact. In the case of frictional contact, velocity and surface traction fields are decomposed:

$$
\begin{aligned}
\underline{\hat{\dot{W}}}_{n+1 / 2}^{E} & =\left(\underline{N}_{E E^{\prime}} \cdot \underline{\hat{\dot{W}}}_{n+1 / 2}^{E}\right) \underline{N}_{E E^{\prime}}+\pi \underline{\hat{\dot{W}}}_{n+1 / 2}^{E} \\
& =\underline{\hat{\dot{W}}}_{n+1 / 2}^{E} \underline{N}_{E E^{\prime}}+\boldsymbol{\pi} \underline{\hat{\hat{W}}}_{n+1 / 2}^{E}
\end{aligned}
$$

where $\underline{N}_{E E^{\prime}}$ is the outward unit normal vector from $\Omega^{E}$ to $\Omega^{E^{\prime}}$.

Contact conditions are: $\forall \underline{M} \in \gamma^{E E^{\prime}}, \forall t \in[0 ; T]:$

$$
\mid \begin{aligned}
& \underline{\hat{F}}_{n+1 / 2}^{E}+\underline{\hat{F}}_{n+1 / 2}^{E^{\prime}}=0 \\
& \underline{\hat{F}}_{n+1 / 2}^{E} \leq 0 \\
& \left(\underline{\hat{W}}_{n+1 / 2}^{E^{\prime} N}-\underline{\hat{W}}_{n+1 / 2}^{E}{ }^{N}\right) \geq j \\
& \left.\underline{\hat{F}}_{n+1 / 2}^{E^{\prime} N}-\underline{\hat{F}}_{n+1 / 2}^{E}{ }^{N}\right)\left(\underline{\hat{W}}_{n+1 / 2}^{E^{\prime} N}-\underline{\hat{F}}_{n+1 / 2}^{E}{ }^{N}-j\right)=0
\end{aligned}
$$

where $j$ is the initial gap.
In the normal direction, we define a local indicator which can be determined with the known element $s_{n}$ :

$$
\begin{aligned}
2 C_{n}(t)= & \frac{\left(\hat{\hat{W}}_{n+1 / 2}^{E^{\prime} N}(t)-\underline{\hat{W}}_{n+1 / 2}^{E^{\prime} N}(t)-j\right)}{(1-\gamma) \Delta t} \\
& +\frac{\left(\hat{\hat{W}}_{n+1 / 2}^{E^{\prime} N}(t)-\underline{\hat{W}}_{n+1 / 2}^{E^{\prime} N}(t)\right)}{k_{0}}
\end{aligned}
$$

the sign of this indicator gives us the solution in the normal direction:

separation: $C_{n}>0$

$\underline{\hat{F}}_{n+1 / 2}^{E}{ }^{N}=\underline{\hat{F}}_{n+1 / 2}^{E^{\prime} N}=0, \quad \underline{\hat{\dot{W}}}_{n+1 / 2}^{E}{ }^{N}=\underline{\dot{W}}_{n+1 / 2}^{E}{ }^{N}-\frac{1}{k_{0}} \underline{F}_{n}^{E N}$,

$\underline{\hat{W}}_{n+1 / 2}^{E^{\prime} N}=\underline{\dot{W}}_{n+1 / 2}^{E^{\prime} N}-\frac{1}{k_{0}} \underline{F}^{E^{\prime} N}$

contact: $C_{n} \leq 0$

$\underline{\dot{F}}_{n+1 / 2}^{E}{ }^{N}=-\underline{\dot{F}}_{n+1 / 2}^{E^{\prime} N}=k_{0} C_{n}$,

$\underline{\hat{W}}_{n+1 / 2}^{E}{ }^{N}=\frac{1}{2}\left\{\underline{\dot{W}}^{E N}+\underline{\dot{W}}^{E^{\prime} N}-\frac{1}{k_{0}}\left(\underline{F}^{E N}+\underline{F}^{E^{\prime} N}\right)\right\}$,

$\underline{\dot{W}}_{n+1 / 2}^{E^{\prime} N}=\underline{\hat{\dot{W}}}_{n+1 / 2}^{E} N$

The Coulomb law is used here. The conditions are

$\begin{cases}\text { if }\left\|\underline{F}_{T}\right\| \leq \mu\left|F_{N}\right| \text { with }\left\|\underline{F}_{T}\right\|<\mu\left|F_{N}\right| & \text { then } \underline{\underline{U}}_{T}=0 \\ \text { if }\left\|\underline{F}_{T}\right\|=\mu\left|F_{N}\right| & \text { then } \exists \lambda>0 \text { satisfying } \underline{\dot{U}}_{T}=-\lambda \underline{F}_{T}\end{cases}$

where $\mu$ is the friction coefficient, $\underline{F}_{T}=\pi \underline{F}$ denotes the tangential part of $\underline{F}$ and $\pi$ denotes the tangential coefficient operator.

In the tangential direction, we define a vectorial indicator:

$$
\begin{aligned}
2 \underline{G}_{n}(t)= & k_{0} \pi\left[\dot{\hat{\hat{W}}}_{n+1 / 2}^{E^{\prime}}(t)-\underline{\hat{\hat{W}}}_{n+1 / 2}^{E}(t)\right] \\
& -\pi\left[\underline{\hat{F}}_{n+1 / 2}^{E^{\prime}}(t)-\underline{\hat{F}}_{n+1 / 2}^{E}(t)\right]
\end{aligned}
$$

This indicator gives the solution, using the sliding limit $g=$ $\mu\left|\underline{\hat{F}}_{n+1 / 2}^{E} \cdot \underline{N}_{E E^{\prime}}\right|$ sticking: $\left\|\underline{G}_{n}\right\| \leq g$

$\pi\left(\underline{\hat{F}}_{n+1 / 2}^{E}\right)=-\pi\left(\underline{\hat{F}}_{n+1 / 2}^{E^{\prime}}\right)=\underline{G}_{n}$,

$\left.\pi \underline{\hat{\dot{W}}}_{n+1 / 2}^{E}=\pi \underline{\dot{W}}_{n}^{E}-\frac{1}{k_{0}} \pi \underline{\left(\hat{F}_{n+1 / 2}^{E}\right.}-\underline{F}_{n}^{E}\right)$

$\pi \underline{\hat{\dot{W}}}_{n+1 / 2}^{E^{\prime}}=\pi \underline{\hat{\dot{W}}}_{n+1 / 2}^{E}$ 

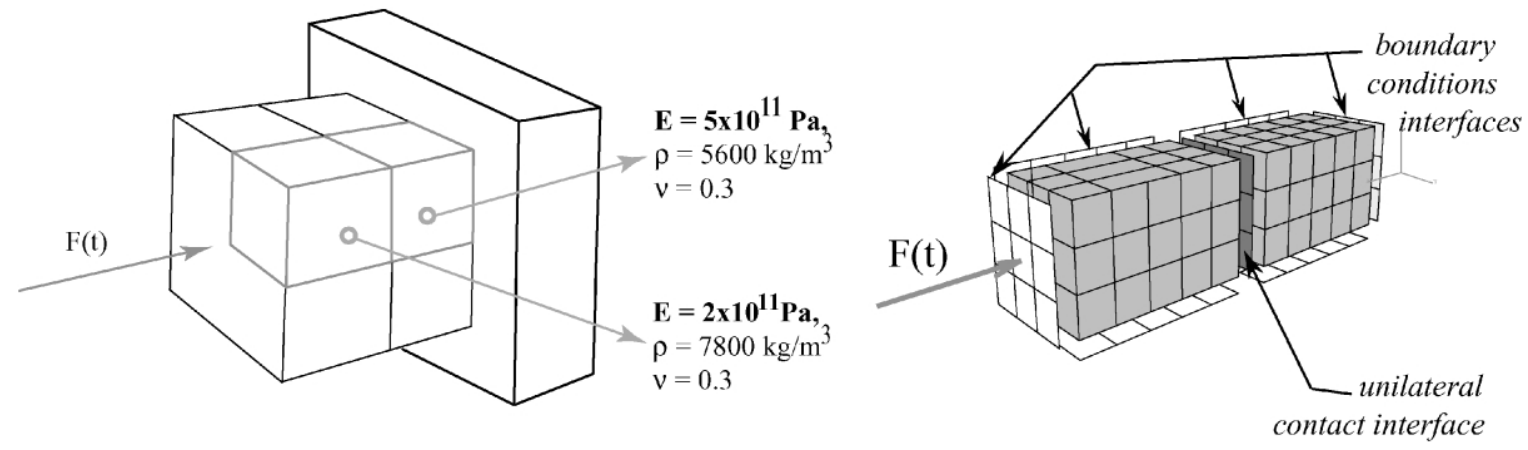

Fig. 4. Two specimens crushing, problem and meso-modelisation.

sliding: $\left\|\underline{G}_{n}\right\|>g$

$\pi\left(\underline{\hat{F}}_{n+1 / 2}^{E}\right)=-\pi\left(\underline{\hat{F}}_{n+1 / 2}^{E^{\prime}}\right)=g \frac{G_{n}}{\left\|G_{n}\right\|}$,

$\pi \underline{\hat{\dot{W}}}_{n+1 / 2}^{E}=\pi \underline{\dot{W}}_{n}^{E}-\frac{1}{k_{0}} \pi\left(\underline{\hat{F}}_{n+1 / 2}^{E}-\underline{F}_{n}^{E}\right)$,

$\pi \underline{\hat{\dot{W}}}_{n+1 / 2}^{E^{\prime}}=\pi \dot{\underline{W}}_{n}^{E^{\prime}}-\frac{1}{k_{0}} \pi\left(\underline{\hat{F}}_{n+1 / 2}^{E^{\prime}}-\underline{F}_{n}^{E^{\prime}}\right)$

Plasticity. We introduce here a classical unidimensional rigid-plastic behaviour. A single internal variable formulation is used. Let $u$ be this variable. The associated thermodynamic force is called $R$. At the initial time $t_{0}, u$ can be negative: $u_{t=0}=u_{0} \leq 0$. This is used, for example, to apply a prestress to a bolt (this point will be detailed further). The evolution of this variable is given by the plastic threshold at time $t$;

$R_{t}=\max _{\tau \leq t}\left(\sigma_{e}, \underline{\hat{F}}_{n+1 / 2}^{E}(\tau)\right):$

if $\underline{\hat{F}}_{n+1 / 2}^{E}(t)<\quad \dot{u}=0$
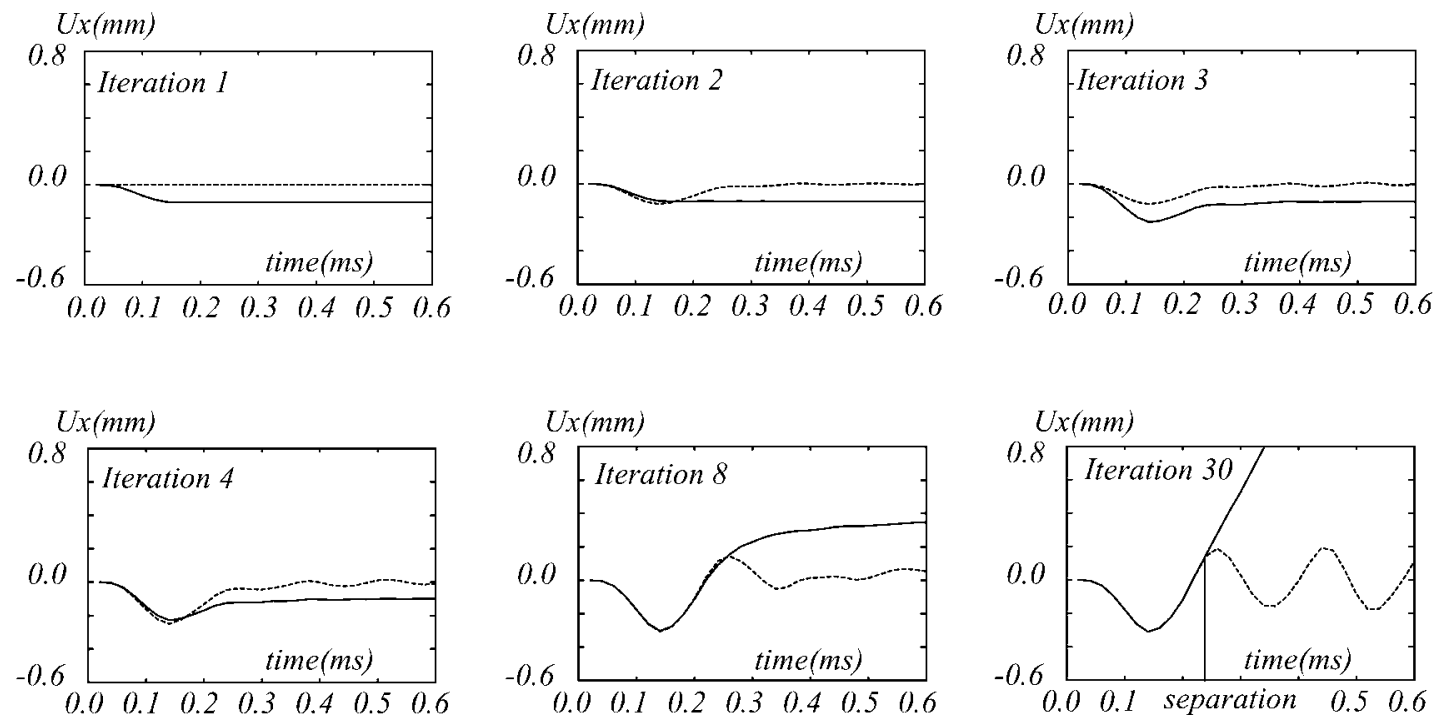

Fig. 5. Evolution in contact during the iterations. 

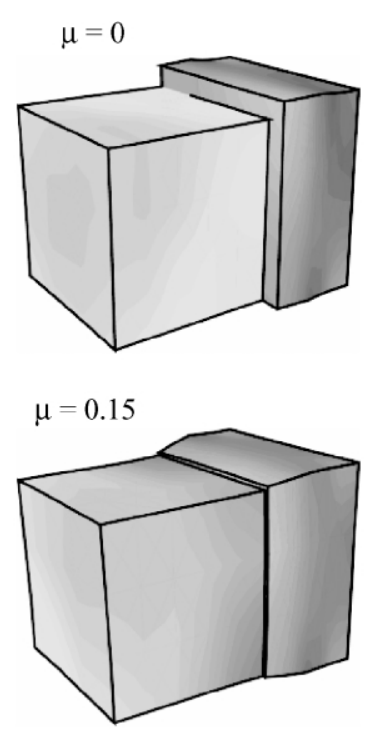
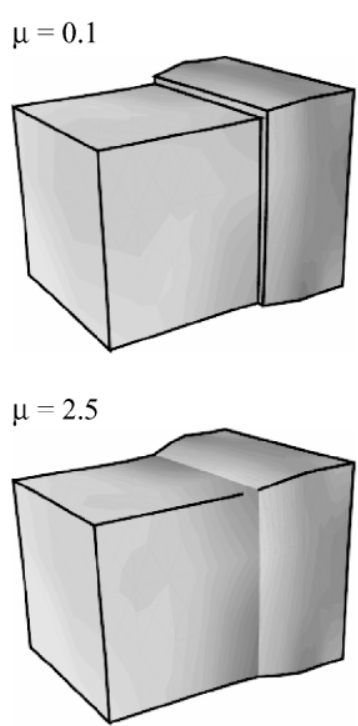

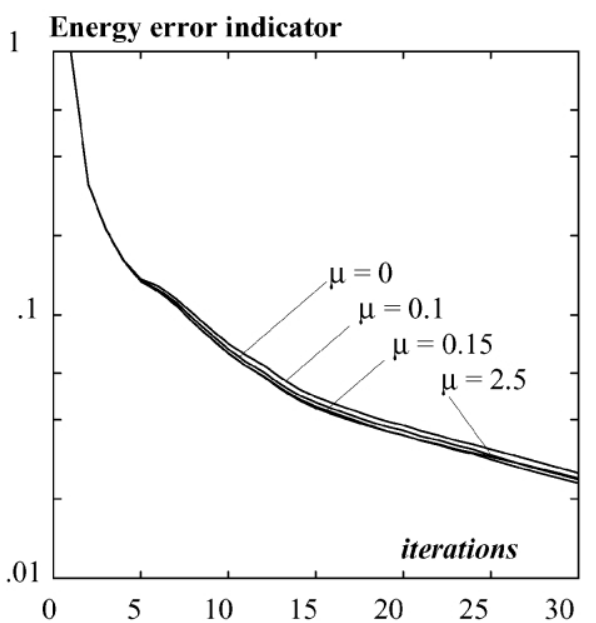

Fig. 6. Influence of sliding coefficient value.

$\underline{\hat{W}}_{n+1 / 2}^{E}(t)=\underline{\dot{W}}_{n}^{E}(t)+\frac{1}{k_{0}}\left(\underline{\hat{F}}_{n+1 / 2}^{E}(t)-\underline{F}_{n}^{E}(t)\right)$,

$\hat{\dot{W}}_{n+1 / 2}^{E^{\prime}}(t)=\underline{\dot{W}}_{n}^{E^{\prime}}(t)+\frac{1}{k_{0}}\left(\underline{\hat{F}}_{n+1 / 2}^{E^{\prime}}(t)-\underline{F}_{n}^{E^{\prime}}(t)\right)$.

This approach has been introduced in a 3D finite element code DYGITA3D, which uses an object oriented method of programming. CASTEM2000 is used to create the mesh and to post-treat the results. An user interface has also been developed to simplify the problem description. We present here two examples: a very simple one to validate and to bring out the characteristics of our approach. The results have been compared with the LS-DYNA3D solutions. The second example quite representative of a real structure assembly (even if the number of d.o.f. is not large) is performed to show the capacity of our approach to solve complex problems.
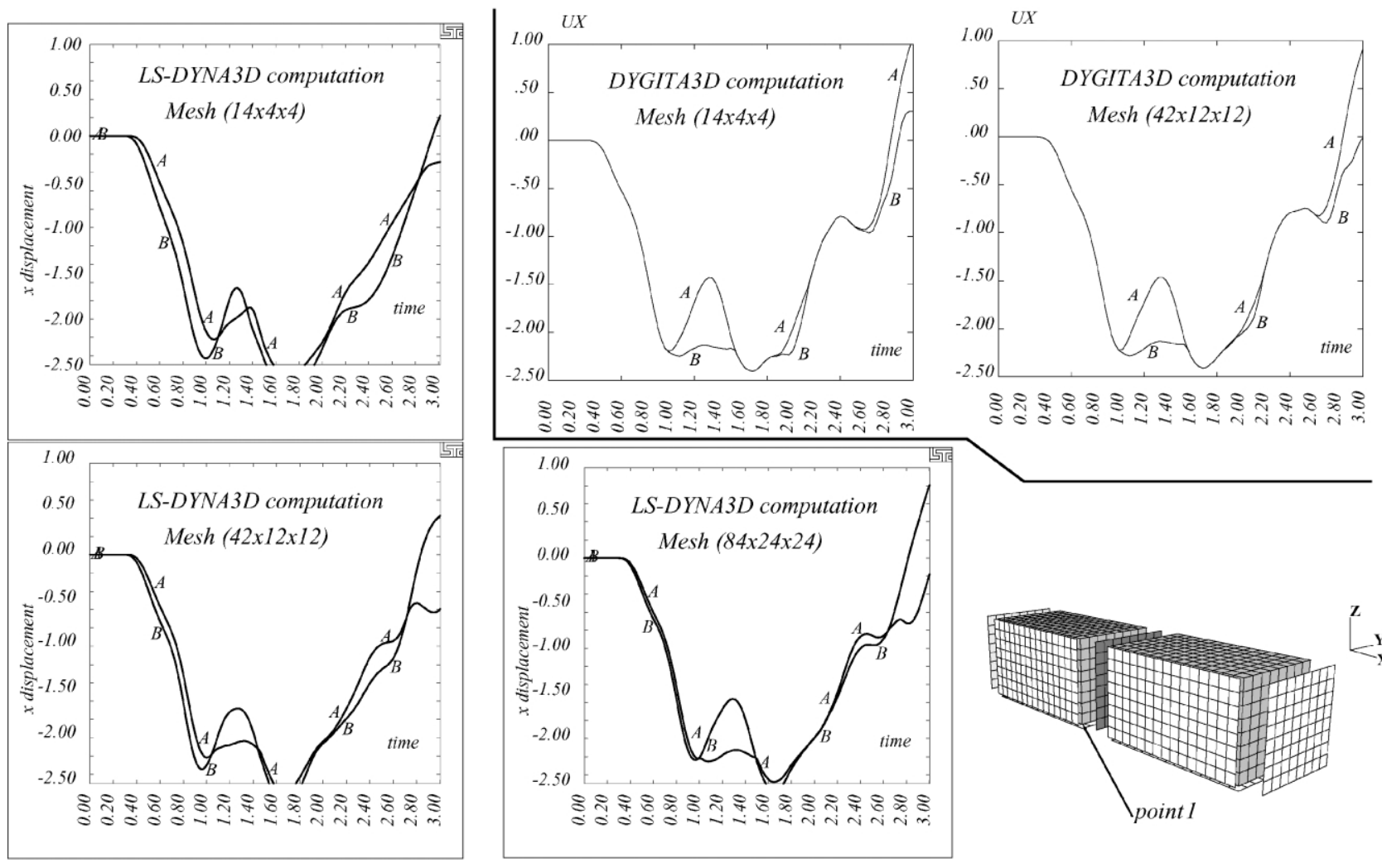

Fig. 7. Axial displacement versus time for two points in contact. 


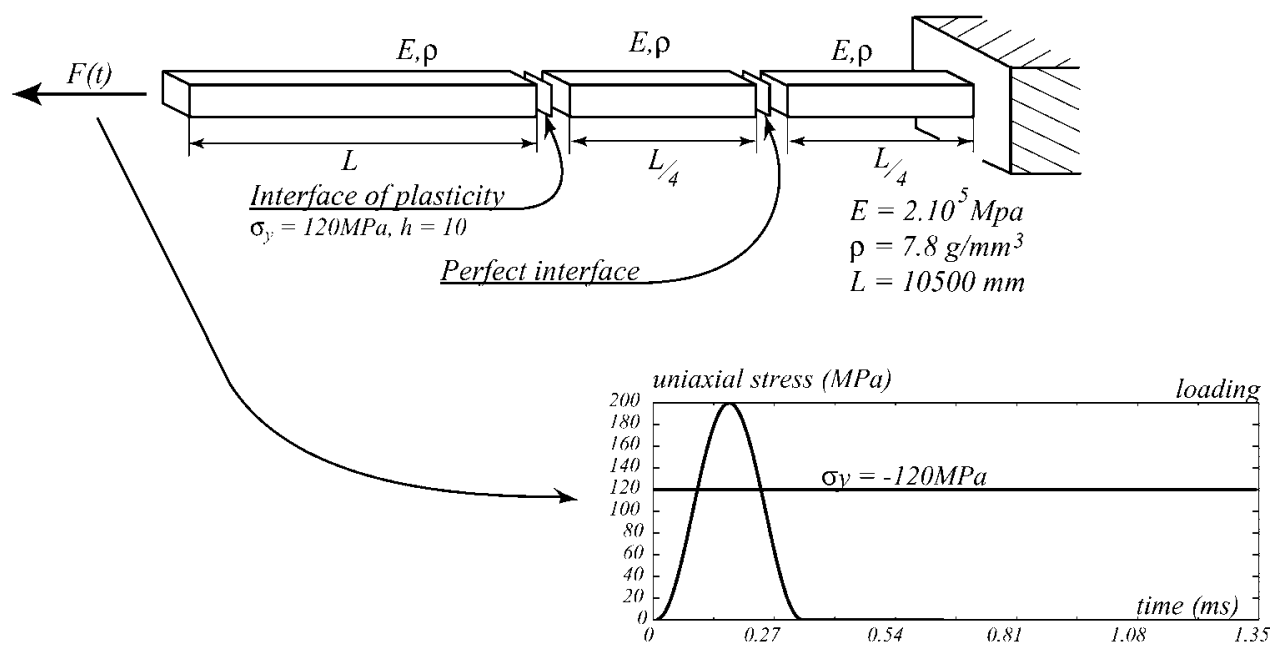

Fig. 8. Structure with an interface of plasticity.

\section{Example one: two specimens crushing}

This example demonstrates both the capacity of the method in treating friction problems and validates our results in terms of solution quality and computational costs.

Two specimens, whose mechanical characteristics are very different, are in contact with a wall. The only non-zero sliding coefficient is between the two sub-structures. Because of the symmetry, only a quarter of the structure needs to be studied. This problem and its corresponding meso-model are shown in Fig. 4.

Since this problem has no analytical solution, the reference then becomes our solution based on a large number of iterations (30 iterations). This solution has been shown in Fig. 5. The central displacement of the interface is plotted for both specimens.

The solid lines represent the hard specimen and the dashed lines represent the soft specimen. The evolution of these curves throughout the method's iterations is also plotted. The method's classical behaviour appears. The plotted quantities are relevant to the sub-structures; thus, they satisfy the contact condition only at convergence, therefore the contact states can be corrected in space as well as in time.

Fig. 6 shows the solution for different values of the sliding coefficient, for the maximum right displaced configuration. From the frictionless to the non-sliding case, several solutions are represented. One can observe from the value of the convergence indicator that the algorithm is very stable for all theses values of friction coefficients. This demonstrates the capacity of the method to solve complex problems with the same ease as the simple frictionless case.

We are now testing the same structure with a new

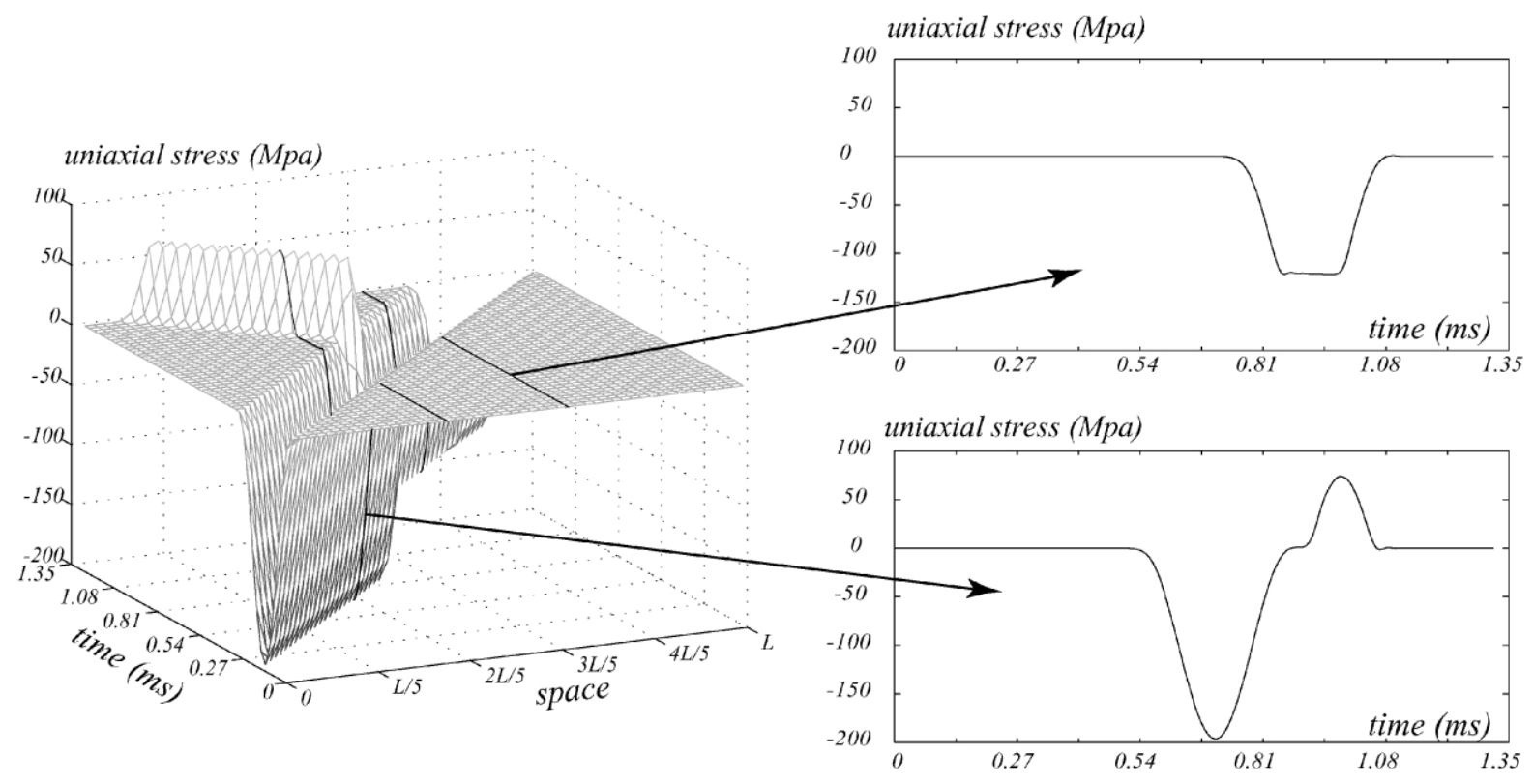

Fig. 9. Uniaxial stress at convergence. 


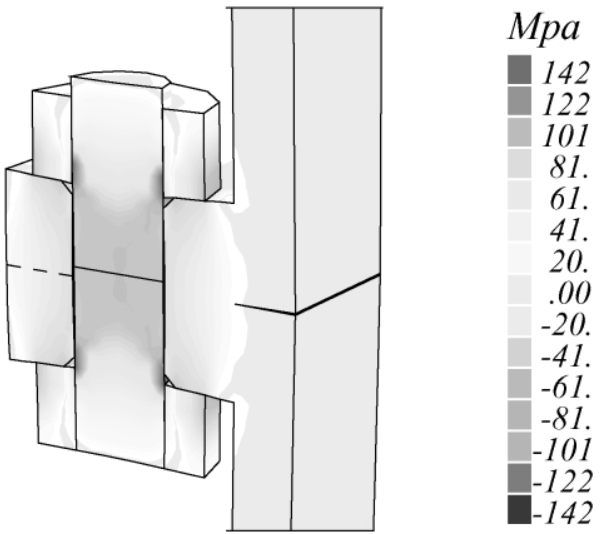

Fig. 10. $\sigma_{z z}$ plotted on the deformed mesh.

loading, associated with a small wavelength compared to the structure size in order to treat a complex dynamic problem. The results are given for different meshes (regular) shown in Fig. 7.

Many time histories of displacement are given from both codes LS-DYNA3D and DYGITA3D. It appears that the solution of this problem is greatly dependent on the contact satisfaction quality. Using penalty formulation to treat contact conditions means that an idea of the solution has to be known in order to choose a good space-time discretisation. Using our approach, even with a poor mesh we obtain a good solution quality and there is no need to have an extremely refined mesh near the interfaces.

Moreover, if the solution quality is not good enough, it can be improved iterating. With classical codes, improving a solution means to make another calculation. To reach the same solution quality the computation times become comparable.

\section{Example two: beam with interface of plasticity}

In order to show the capacity of the method in representing plasticity problems, a simple example is presented.
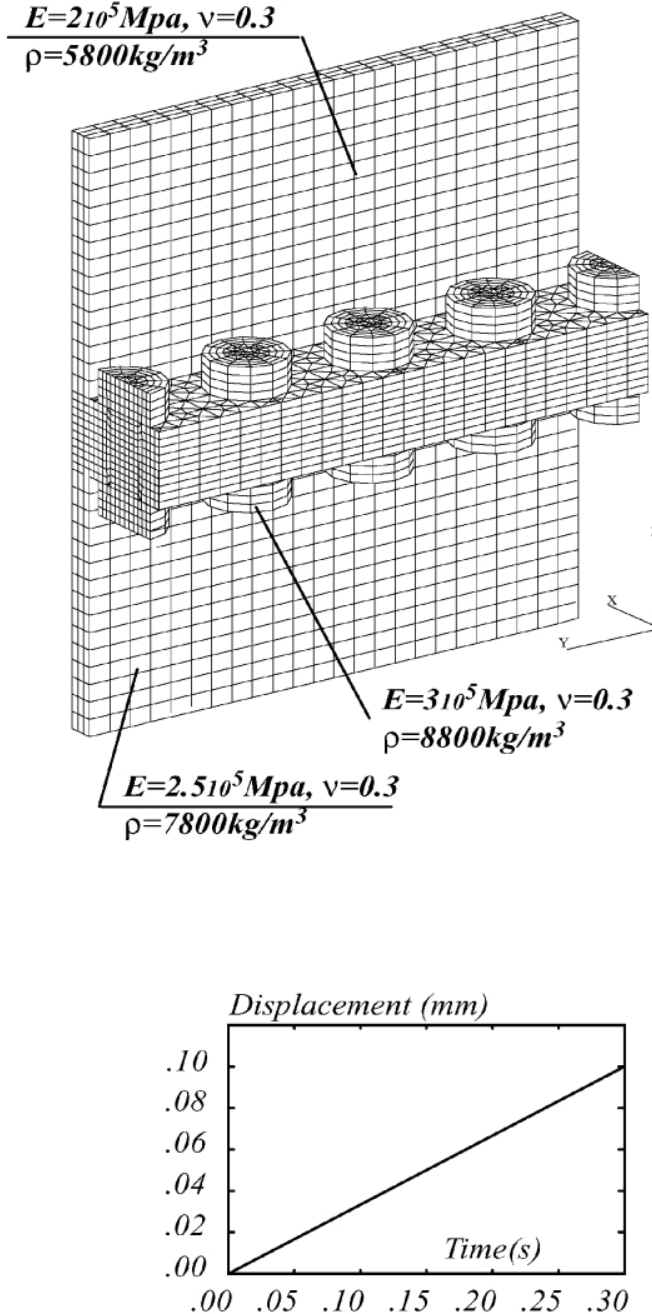

Loading for prestress of the bold
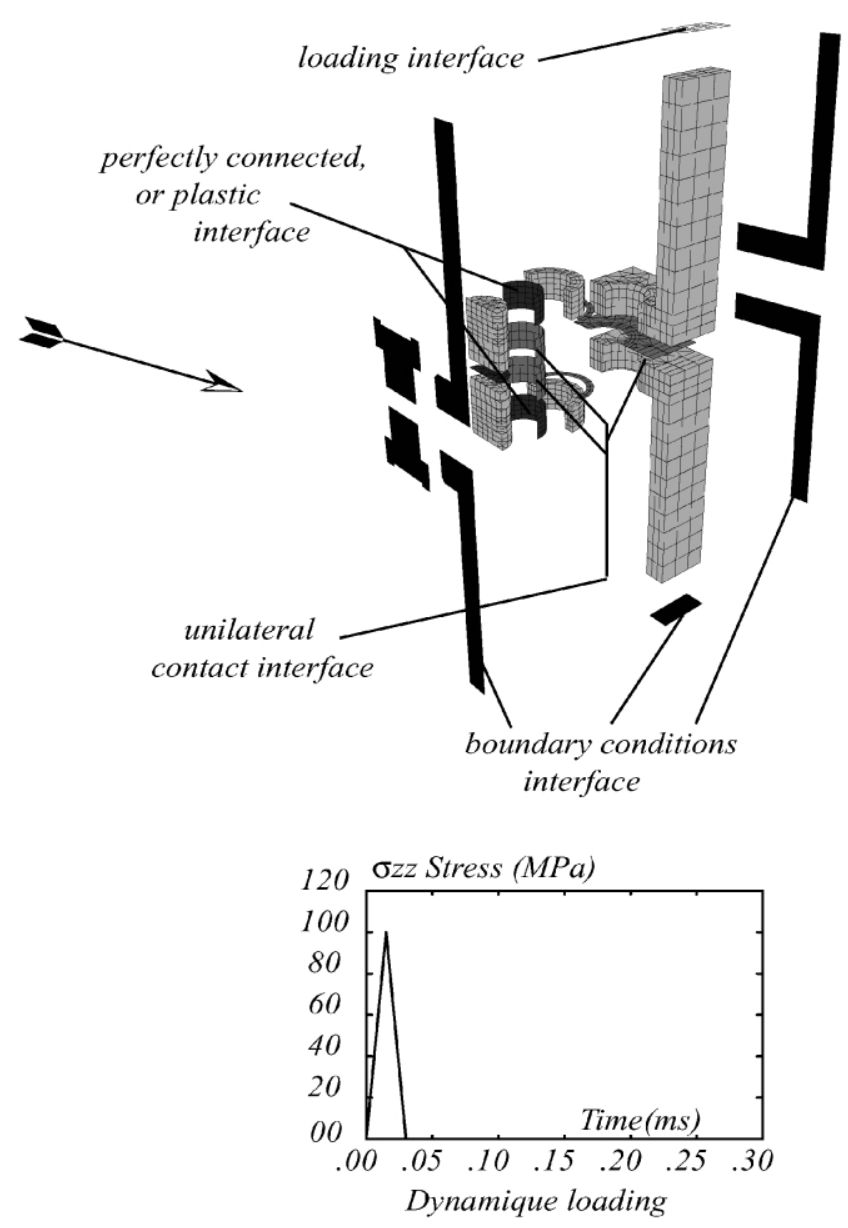

Fig. 11. Simple bolting assembly. 
DYGITA3D computation

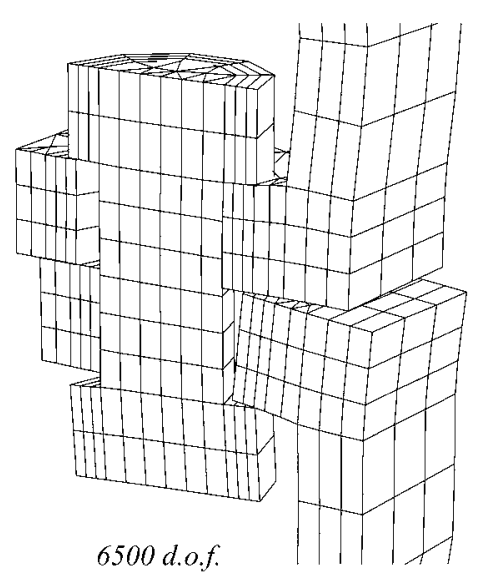

DYNA-3D computation

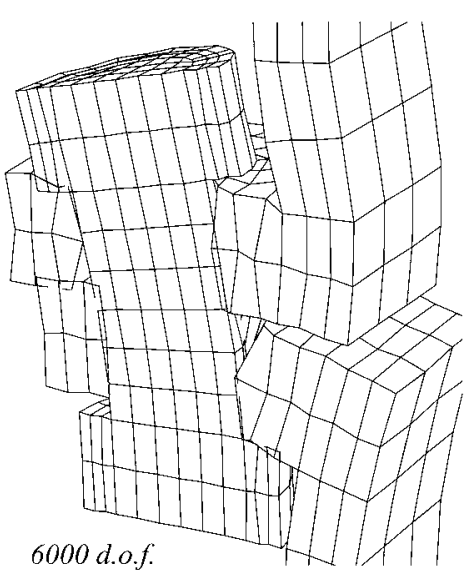

DYNA-3D computation

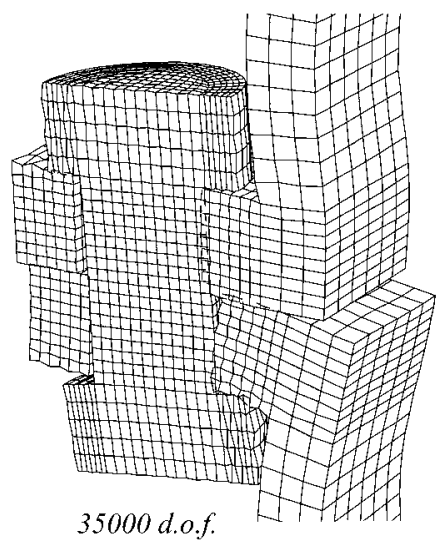

Fig. 12. Comparison of deformed mesh from LS-DYNA3D and DYGITA3D computations.

The structure is shown in Fig. 8, its meso-modelisation is composed of three sub-structures with a perfect interface and an interface of plasticity. The elastic limit of $120 \mathrm{MPa}$ is depicted on the time loading in Fig. 8.

It is important to notice that an interface of plasticity is used because it fits well the description of the non-linearities, all taken into account at the interfaces. This description is an approximation of the real structure behaviour, but it is sufficient to represent the major phenomena occurring during the two-stages separation of a launcher.

The solution is shown in Fig. 9. It shows the rebound of the wave when its level reach the elastic limit. In fact this interface acts like a perfect interface when the elastic limit is not reached and as a free interface beyond this limit. It is important to notice here that convergence results for this computation are the same as those obtained in the case of frictional contact. It means that taking into account bolt's plasticity does not involve much more difficulties in the computation.

\section{Example three: bolted structure}

The studied structure is composed of two flanges bolted together. This structure and its meso-modelisation are shown in Fig. 10.

Two different loadings are applied. The first one is used to pre-stress the bolt and the second one is a dynamic loading pulling the top interface. The time scale of the two loadings is very different, even though the same code DYGITA3D is used for both. This is permitted because of the use of an implicit time integration scheme.

The solution of the first computation is shown in Fig. 11. The pre-stress of the bold is applied using the interface that links the rod and the top head of the bolt and by prescribing a negative displacement of $0.06 \mathrm{~mm}$. Only two time steps are used to obtain this solution. Furthermore, the computational time associated with this calculation is negligible compared to the dynamic one and using the same code to prescribe the pre-stress bolt is a big advantage.
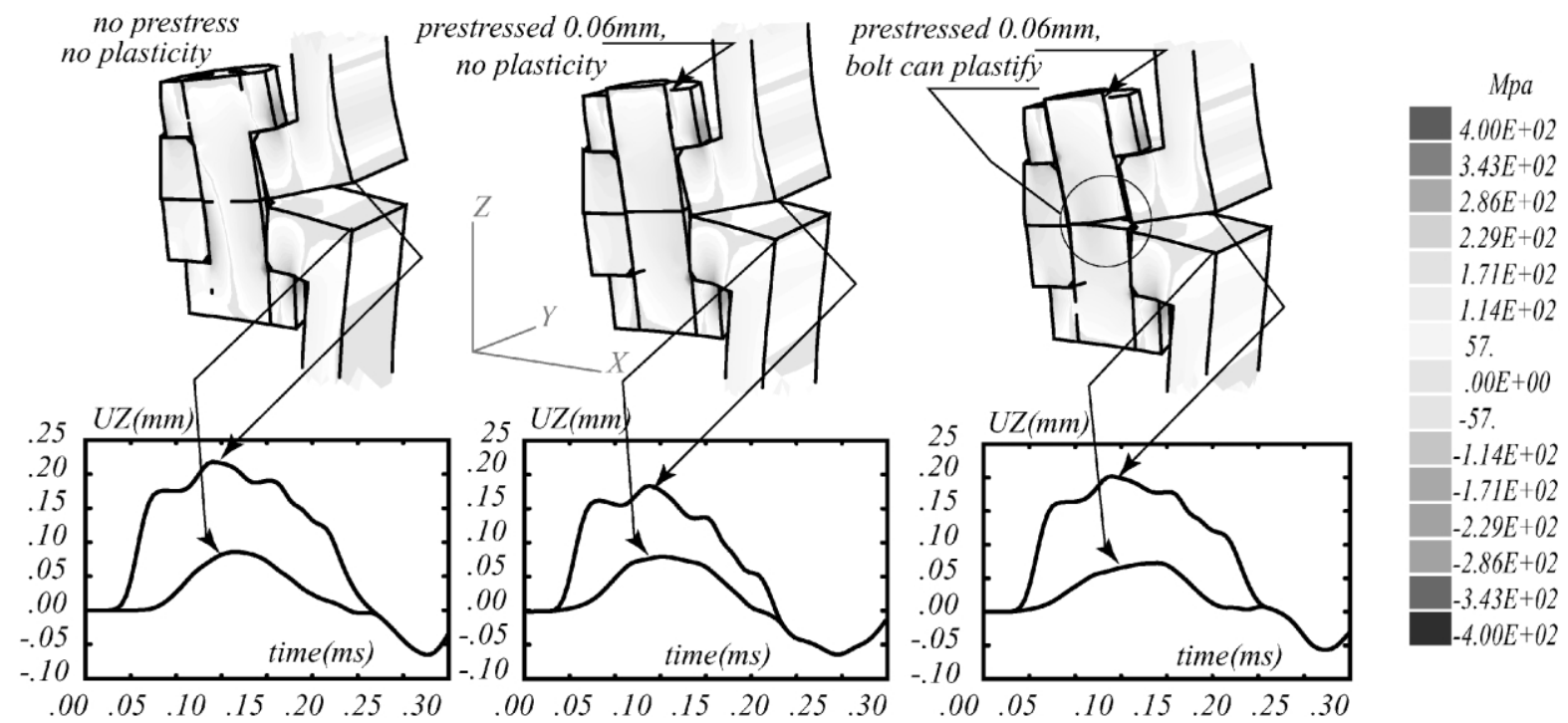

Fig. 13. Comparison, taking into account pre-stress and/or plasticity. 
We now compare the LS-DYNA3D and the DYGITA3D computations by taking into account only the dynamic loading.

It is obvious that the solutions are similar and that a very fine mesh is necessary for LS-DYNA3D to obtain the solution. Therefore, the computational times associated with a solution quality are comparable (about $30 \mathrm{~min}$ CPU for 6500 d.o.f. and 200 time steps on a standard workstation). However, for very large scale problems, the computational time for LS-DYNA3D seems to be much larger.

All the ingredients needed to represent a real shock propagation through an assembly are integrated in our code DYGITA3D. We finally group them in a computation in order to show the feasibility of studying the influence of the pre-stress of the bolts and the introduction of the bolt rod's plasticity (Fig. 12).

These behaviours are plotted in Fig. 13 and demonstrate the capacity of our approach by treating real propagation of a shock through an assembly.

The CPU time associated with these computations is about 30 min which is very encouraging for studying large number of freedom problems.

\section{Conclusion}

A new approach for solving dynamic problems in threedimensional complex structures of assembly, submitted to shocks has been presented herein. It is the continuation of previous works [1-3,7] and all ingredients are now introduced to represent the effect on a shock response spectrum of a real shock passing by a assembly (contact, friction, plasticity). Our approach is based on two components: a mixed decomposition of the structure which provides significant modularity to the problem description; and an iterative solution scheme that is welladapted to the problem.

The numerical results in the case of elastic problems have demonstrated its capability in solving frictionalcontact problems and plasticity problems with the same degree of difficulty.

Moreover, the special treatment of contact conditions and plasticity, and the resolution technique used here, involving constant operators throughout the iterations make this approach suitable for 3D computations on complex structures.

These results will help to establish shock response spectrum of real pyro-impacts or condensed constitutive law of an assembly, and by doing so will help to explain real effects of these shocks on the structure. Furthermore, new studies using in particular the natural parallel characteristic of the method are forthcoming to reduce the computational costs in order to treat real structure of complex assemblies.

\section{References}

[1] Blanzé C, Champaney L, Cognard JY, Ladevèze P. A modular approach to structure assembly computations, application to contact problems. Engng Comput 1996;13(1):15-32.

[2] Boucard PA, Ladevèze P, Lemoussu H. A modular approach to 3-D impact computation with frictional contact. Comput Struct; 78 (1): 45-52.

[3] Champaney L, Cognard JY, Dureisseix D, Ladevèze P. Large-scale application on parallel computers of a mixed decomposition method. Comput Mech 1997;19:253-63.

[4] Hallquist JO, Goudreau GL, Benson DJ. Sliding interfaces with contact-impact in large-scale Lagrangian computations. Comput Meth Appl Mech Engng 1985;51:107-37.

[5] Hoff C, Hughes TJR, Hulbert G, Pahl PJ. Extended comparison of the Hilbert-Hughes-Taylor $\alpha$-method and the $\theta$-method. Int $\mathbf{J}$ Numer Meth Engng 1989;76:87-93.

[6] Ladevèze P. Sur une famille d'algorithmes en mécanique des structures. C R Acad Sci Paris 1985;II:41-4.

[7] Ladevèze P. Non-linear computational structural mechanics. New York: Springer; 1998. French version 1996.

[8] Lions PL. On the Schwarz alternating method III. A variant of nonoverlapping sub-domains. In: Chan T, Glovinski R, Priau J, Windlum O, editors. Proceedings of Domain Decomposition Methods for Partial Differential Equations. Philadelphia: SIAM; 1990.

[9] Newmark NM. A method of computation for structural dynamics. J Engng Mech Div 1959;EM3:67-94.

[10] Verpeaux P, Charras T, Millard A. CASTEM2000 une approche moderne du calcul des structures. In: Fouet JM, Ladeveze P, Ohayon R, editors. Calcul des Structures et Intelligence Artificielle. Pluralis; 1988. p. $261-71$.

[11] Zienkiewicz OC, Taylor RL. The finite element method, 4th ed. London: McGraw-Hill; 1991. 